\title{
Comparative study of teleophthalmology devices: Smartphone adapted ophthalmoscope, robotic ophthalmoscope, and traditional fundus camera - The recent advancements in telemedicine
}

\author{
James B. Aguayo Martel ${ }^{1-4}$, Ursula M. Anders ${ }^{1,2}$ and Victoria Kravchuk ${ }^{1,2}$ \\ ${ }^{1}$ Martel Eye Medical Group, Rancho Cordova, CA, USA \\ ${ }^{2}$ Leading Medical Technologies, Inc. Rancho Cordova, CA, USA \\ ${ }^{3}$ California Northstate University College of Medicine, Elk Grove, CA, USA \\ ${ }^{4}$ Dignity Health, Carmichael, CA, USA
}

Teleophthalmology provides specialist consultation services in places where they are not readily available. It has created an alternative mechanism to which patients in remote or underserved regions of the nation can have access to expert opinion and evaluation. Certain remote locations are carefully selected to hold stationary devices that would allow for funduscopic images to be obtained. Images are then transferred to a reading center where an ophthalmologist reviews them and decides on the diagnosis and treatment options for the patient. During the initial development of this system, a great deal of effort was put into analyzing image quality in order to standardize the reading of images [1]. Initial studies analyzed the progression of diabetic retinopathy for the benefit and modality of treatment for diabetes. This culminated in diabetic screening and treatment protocols $[2,3]$. These studies proved that large populations of patients with certain diseases could be followed, and that the data gathered from these studies could be used to define different conditions and stages of diseases. The use of teleophthalmology has also proven to be effective in the areas of glaucoma and retinopathy of prematurity, among other retinal diseases $[4,5]$.

The fields of telemedicine and teleophthalmology have greatly expanded both in their scope and prevalence over the past 20 years. Telemedicine was first introduced in the area of cardiology in 1989 [6] and has since undergone rapid development and widespread adoption in many areas of medicine. It enabled physicians to extend their capabilities in situations where they would not have been able to do so previously. In 1990, a mobile cellular version of telemedicine was released [6]. The use of cellular devices has become increasingly popular in the medical field. A 2010 survey showed that ninty nine percent of health professionals had mobile phones, of which eighty one percent were smartphones [7]. Currently, portable smartphones with attached devices capable of taking funduscopic photographs have shown widespread usage. Applications on smartphones allow for patient education and testing of visual acuity, color vision, Amsler grid, astigmatism, and contrast sensitivity. The field of robotics has also experienced a rapid growth in technology and is now being utilized as a platform by which ophthalmoscopy or ocular exams can be performed. This advanced technology enables live-stream and store-and-forward imaging with an ophthalmoscope attachment. With mobile platforms, the use of teleophthalmology can allow direct one on one feeds between the physician and patient.

There is a current need for emergency teleophthalmology services in remote and underserved areas [8-9]. Hospitals and clinics that do not have access to ophthalmologists may lead to patients being improperly diagnosed or improperly transferred to another facility where an ophthalmologist is available for consult. A way to avoid this would be to integrate mobile teleophthalmology services in these areas. Emergency teleophthalmology services were used to identify urgent cases at a hospital in Brazil [10]. Eighty-five percent of patients were correctly identified as being urgent or nonurgent cases. Two percent of patients were misinterpreted as non-urgent cases and thirteen percent were misinterpreted as urgent cases. The limitation of the study was that the images were taken with a cellphone and +60 diopter lens, which was better suited for detecting anterior segment diseases. A recent comparison of technical specifications was made between several handheld and smartphone-based fundus cameras [11]. However the issues of utilization and image quality were not addressed. This study compares two of these mobile systems (Welch-Allyn Panoptic and JEDMED Horus Scope attached to an InTouch Lite robotic system) compared to a traditional in-office fundus photography system (Zeiss Visucam NM/FA), with the intention of determining the feasibility of integrating emergency teleophthalmology services into more rural

Correspondence to: Dr. James Benjamin AguayoMartel, MD MPH FACS, Clinical Professor of Ophthalmology, California Northstate University College of Medicine, 9700 W Taron Drive, Elk Grove, CA 95757, Tel: (916) 6867300, Fax: (916) 6867310, E-mail: james.martel@cnsu.edu

Keywords: telemedicine, teleanalytics, teleophthalmology, remote care, emergency care, intensive care, smartphone

Received: October 04, 2015; Accepted: October 09, 2015; Published: October 13, 2015 
Martel J (2015) Comparative study of teleophthalmology devices: Smartphone adapted ophthalmoscope, robotic ophthalmoscope, and traditional fundus camera The recent advancements in telemedicine

clinics and hospitals.

\section{Materials and methods}

\section{Instruments}

The remote modalities include the Welch-Allyn Panoptic 11820 (Welch-Allyn, Skaneateles Falls, NY, USA) attached to an Iphone 4 (Apple Inc., Cupertino, CA, USA), and a JEDMED HD digital camera with a Horus Scope fundus attachments (Jedmed Instrument Co, St. Louis, MO, USA). The JEDMED system was linked to an InTouch Lite robot (InTouch Technologies, Inc., Santa Barbara, CA, USA). The stationary fundus camera used as the control was a Zeiss Visucam NM/ FA (Carl Zeiss AG, Oberkochen, Germany).

\section{Remote Capabilities}

Images from the Visucam could be seen from an in-network VNC viewer (RealVNC Limited, Cambridge, UK) enabled from a desktop computer or Ipad. They could also be exported and sent via HIPAA secured email or mail. Images from the Horus Scope could be seen from the InTouch Health HIPAA secure application on a smartphone or desktop. Images from the Welch-Allyn could be sent from a HIPAA secure application on the Iphone, such as OnePass (Emerge MD Inc., Phoenix, AZ, USA).

\section{Methods}

Examinations were conducted in an ophthalmology clinic over a period of one year. The patients were dilated with cycloplegics and images from all three modalities were obtained by an experienced ophthalmic technician. All patients received subsequent indirect ophthalmoscopic exams from an ophthalmologist to correlate the findings.

\section{Results}

The three modalities were compared in terms of portability, resolution, price, stereoscopic capability, anterior segment capability, ease of use, image quality, field of view, live stream capability, and store and forward capability (Table 1).

With careful analysis, the readability of the JEDMED system was very close to that which was obtained by the Zeiss system (Figure 1). After some training, the device was manageable to use. There were no circumstances where information that was lost was felt to be critical to the study. A larger field of view of the fundus could be obtained by taking multiple images and collecting them with a collage program. This made for high resolution imagery and the ability to detect small defects (Figure 2). It was also adequate in capturing external photographs (Figure 3). One of the features of the JEDMED is that it could be attached to the robotic InTouch Lite system. It was the more favorable candidate, since it would allow for a full livestream interview with patients so as to give the doctor a more holistic view of the medical problems that the patient had, not just an internal view of the patient's fundus. As far as the issue of expenditure, the JEDMED is significantly more expensive than the Welch-Allyn.

The initial exposure to the Welch-Allyn was useful but there were distinct limitations. It was adequate in training students on how to use an ophthalmoscope, however its utility was limited by the resolution of the camera. The field of vision oftentimes limited the visibility of the retina, and the image was too pixelated in the key principle locations (Figure 2). It did prove useful as an anterior segment device looking for small features in the cornea, anterior chamber, or conjunctiva (Figure 4). It was also difficult to operate the handheld - trying to stabilize the instrument while obtaining clear focus for capturing the image. It has great potential, however a much higher resolution is needed in order for it to become more acceptable.

Both instruments were able to detect a great deal of pathology from both the anterior segment and the retina. Images obtained from the devices resulted in diagnoses of corneal ulcer, corneal opacity, corneal edema, corneal abrasion, recurrent corneal erosion, herpes zoster keratitis, corneal foreign body, bacterial conjunctivitis, viral conjunctivitis, episcleritis, hyphema, hypopyon, synechiae, vitreous hemorrhage, choroidal nevus, Hollenhorst plaque, hypertensive retinopathy, central retinal artery occlusion, central retinal vein occlusion, branch retinal artery occlusion, branch retinal vein occlusion, diabetic retinopathy, hard exudates, dot and blot hemorrhages, flame hemorrhages, retinal hemorrhages secondary to anemia, drusen, retinal edema, histoplasmosis, macular degeneration, anterior ischemic optic neuropathy, traumatic optic neuropathy, papilledema, optic neuritis, and optic nerve cupping.

\section{Discussion}

The development and utilization of telemedicine devices has

Table 1. Comparison of the Zeiss Visucam NM/FA, JEDMED Horus Scope, and Welch-Allyn Panoptic 11820 [4-12].

\begin{tabular}{|l|c|c|c|}
\hline & Zeiss Visucam NM/FA & JEDMED Horus Scope & Yes \\
\hline Portable & No & $1920 \times 1080$ pixels \\
\hline Resolution & $1280 \times 1024$ pixels & $\$ \$$ & Yes \\
\hline Price & $\$ \$ \$$ & No & Yes \\
\hline Stereo Capability & Yes & No & Slightly Difficult \\
\hline Anterior Segment Capability & Easy & Very Good \\
\hline Ease of Use & Excellent & Difficult \\
\hline Image Quality & 30,45 degrees & Pegrees \\
\hline Field of View & No & Yes \\
\hline Live Stream Capability & Yes & Yes \\
\hline Store and Forward Capability & Noor & No \\
\hline
\end{tabular}


Martel J (2015) Comparative study of teleophthalmology devices: Smartphone adapted ophthalmoscope, robotic ophthalmoscope, and traditional fundus camera The recent advancements in telemedicine

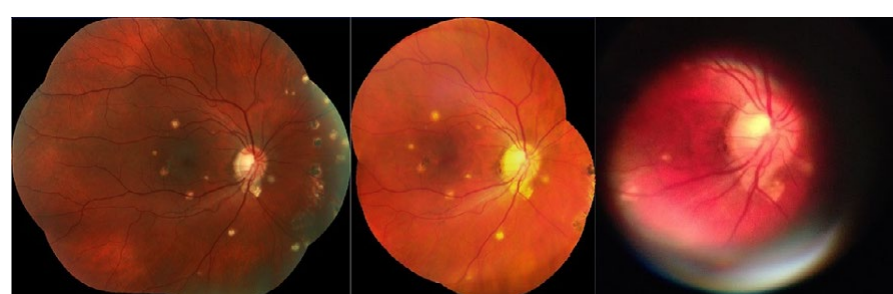

A comparison of the funduscopic images taken of a patient diagnosed with Histoplasma capsulatum retinitis with the Zeiss (left), JEDMED (middle), and Welch-Allyn (right) cameras.

Figure 1. Comparison of funduscopic images.

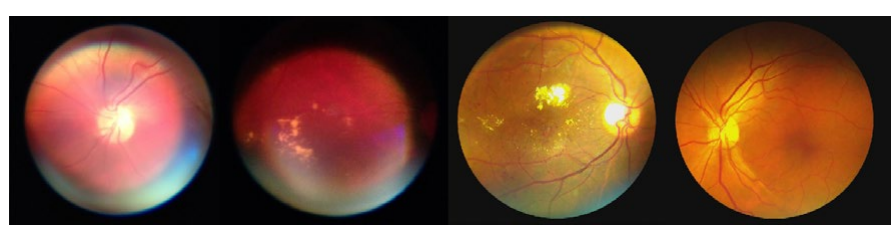

A comparison of the funduscopic images taken with the Welch-Allyn (left) and JEDMED (right) cameras.

Figure 2. Comparison of funduscopic images.

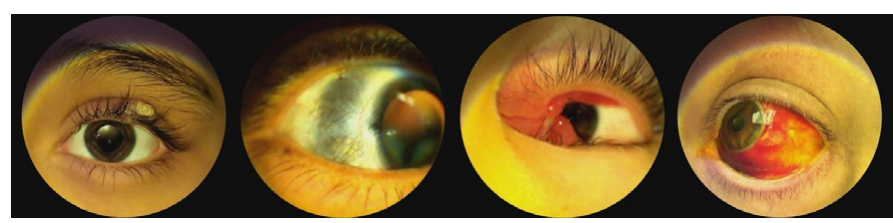

Figure 3. External images taken with the JEDMED Horus Scope.

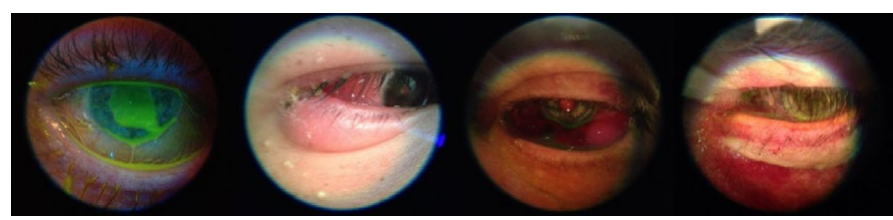

Figure 4. External images taken with the Welch-Allyn Panoptic 11820.

rapidly evolved over the past two decades. The explosive growth of technological development in telecommunications and optoelectronics has culminated in the ability of synchronous telemedicine capabilities from a wireless device to a central viewing site or monitoring service. There are many diseases that teleophthalmology shows great usefulness for, especially in the area of the retina. Current teleophthalmology services are successful for the detection and early intervention for diabetic retinopathy [3], however teleophthalmology services have the capability of extending beyond this. The extension of evaluation to the anterior portion of the eye has been of great utility. The disease entities that can be monitored have greatly expanded from internal diseases to abnormalities involving the cornea, conjunctiva, iris, anterior chamber, and lens.

The evaluation of both the anterior and posterior portions of the eye is useful in emergent and urgent settings. The emergency department evaluates many ocular injuries and illnesses such as chemical burns, corneal ulcers, iritis, optic neuritis, papilledema, retinal artery or vein occlusions, vitreous hemorrhage, traumatic optic neuropathy, macular degeneration, epiretinal membranes, or glaucoma. If an ophthalmologist is not present at the hospital, many of these patients get referred for ophthalmologic follow up at an alternate location. Of these patients, the amount that are actually seen is difficult to assess. An evaluation of these numbers were determined from a trauma center in Brooklyn, NY between June 2003 to October 2005 [15]. Sixty percent of emergency patients, fifty-seven percent of trauma patients, and sixty-six percent of nontrauma patients followed up with an ophthalmologist. Teleophthalmology services in cases such as these would allow for immediate detection and treatment of ocular injuries and diseases, which would ultimately improve patient outcomes.

In order to assist the physician with making the correct diagnosis, it is crucial for the equipment to be easy to use, reliable, and accurate. Of the mobile devices that were tested, the JEDMED attached to the InTouch Lite robotic system has overall better function than the Welch-Allyn. The JEDMED is easier to use, has live-stream capabilities, and offers higher resolution. The ability to connect directly to remote patients allows the ophthalmologist to obtain a complete history and physical in addition to being able to view the funduscopic and anterior segment images. Therefore, this device seems to be the most promising in its ability to extend into more hospitals that have robot capabilities. The Welch-Allyn is a suitable option for the clinical setting, as it is less expensive and can be easily modified. The issue of pixelation can be solved with a higher resolution camera opposed to the Iphone 4's 5 megapixels. Livestream consultations can be accomplished via a HIPAA secured online video feed and VNC connection between clinics. These adjustments would create a suitable device for the delivery of specialty healthcare to more remote clinics.

\section{References}

1. Cavallerano JD, Silva PS, Tolson AM, Francis T, Tolls D, et al. (2012) Imager evaluation of diabetic retinopathy at the time of imaging in a telemedicine program. Diabetes Care 35: 482-484. [Crossref]

2. Silva PS, Cavallerano JD, Aiello LM, Aiello LP (2011) Telemedicine and diabetic retinopathy: moving beyond retinal screening. Arch Ophthalmol 129: 236-242. [Crossref]

3. Li HK, Horton M, Bursell SE, Cavallerano J, Zimmer-Galler I, et al. (2011) Telehealth practice recommendations for diabetic retinopathy, second edition. Telemed J E Health 17: 814-837. [Crossref]

4. Thomas SM, Jeyaraman MM, Hodge WG, Hutnik C, Costella J, et al. (2014) The effectiveness of teleglaucoma versus in-patient examination for glaucoma screening: systematic review and meta-analysis. PLoS One 9: e113779. [Crossref]

5. Wang SK, Callaway NF, Wallenstein MB, Henderson MT, Leng T, et al. (2015) SUNDROP: six years of screening for retinopathy of prematurity with telemedicine. Can J Ophthalmol 50: 101-106. [Crossref]

6. Blyth, WJ. Telecommunications, Concepts, Development, and Management. 2nd edition. Glencoe/McGraw-Hill. 1990: 280-2.

7. Bastawrous A, Cheeseman RC, Kumar A (2012) iPhones for eye surgeons. Eye (Lond) 26: 343-354. [Crossref]

8. Rosengren D, Blackwell N, Kelly G, Lenton L, Glastonbury J (1998) The use of telemedicine to treat ophthalmological emergencies in rural Australia. $J$ Telemed Telecare 4 Suppl 1: 97-99. [Crossref]

9. Kulshrestha M, Lewis D, Williams C, Axford A (2010) A pilot trial of teleophthalmology services in north Wales. J Telemed Telecare 16: 196-197. [Crossref]

10. Ribeiro AG, Rodrigues RA, Guerreiro AM, Regatieri CV (2014) A teleophthalmology system for the diagnosis of ocular urgency in remote areas of Brazil. Arq Bras Oftalmol 77: 214-218. [Crossref]

11. Panwar N, Huang P, Lee J, Keane PA, et al. (2015) Fundus Photography in the 21st Century-A Review of Recent Technological Advances and Their Implications for Worldwide Healthcare. Telemed J E Health. [Crossref]

12. Retrieved from: http://www.zeiss.com/meditec/en_us/products---solutions/ ophthalmology-optometry/retina/diagnostics/fundus-imaging/visucam-nm-fa. html\#highlights (accessed on October 1, 2015) 
Martel J (2015) Comparative study of teleophthalmology devices: Smartphone adapted ophthalmoscope, robotic ophthalmoscope, and traditional fundus camera The recent advancements in telemedicine

13. Retrieved from: http://www.jedmed.com/products/fundus-ophthalmoscope-lens (accessed on October 1,2015)

14. Retrieved from: http://www.welchallyn.com/en/products/categories/physical-exam/ eye-exam/ophthalmoscopes--wide-view-direct/panoptic_ophthalmoscope.html (accessed on October 1,2015).

15. Rizzuti AE, Vastardi M, Hajee M, Lazzaro DR (2013) Scope of resident ophthalmology consultation service and patient follow-up rates at a level 1 trauma center in Brooklyn, New York. Clin Ophthalmol 7: 643-647. [Crossref]

Copyright: (C2015 Martel J. This is an open-access article distributed under the terms of the Creative Commons Attribution License, which permits unrestricted use, distribution, and reproduction in any medium, provided the original author and source are credited. 